\title{
Moments of the Generalized Hyperbolic Distribution
}

\author{
David J Scott*, \\ Department of Statistics, \\ University of Auckland, \\ PB 92019, \\ Auckland, New Zealand, \\ d.scott@auckland.ac.nz \\ Diethelm Würtz, \\ Institut für Theoretische Physik, \\ HIT K32.3, \\ Wolfgang-Pauli-Strasse 27 \\ 8093 Zürich, Switzerland \\ wuertz@phys.ethz.ch \\ Christine Dong, \\ Department of Statistics, \\ University of Auckland, \\ PB 92019, \\ Auckland, New Zealand, \\ c.dong@auckland.ac.nz \\ and \\ Thanh Tam Tran, \\ Department of Statistics, \\ University of Auckland, \\ PB 92019, \\ Auckland, New Zealand \\ ttra021@stat.auckland.ac.nz
}

December 9, 2009 


\begin{abstract}
In this paper we demonstrate a recursive method for obtaining the moments of the generalized hyperbolic distribution. The method is readily programmable for numerical evaluation of moments. For low order moments we also give an alternative derivation of the moments of the generalized hyperbolic distribution. The expressions given for these moments may be used to obtain moments for special cases such as the hyperbolic and normal inverse Gaussian distributions. Moments for limiting cases such as the skew hyperbolic $t$ and variance gamma distributions can be found using the same approach.
\end{abstract}

Keywords: Generalized hyperbolic distribution; hyperbolic distribution; kurtosis; moments; normal inverse Gaussian distribution; skewed- $t$ distribution; skewness; Student- $t$ distribution. 


\section{Introduction}

The generalized hyperbolic distribution was introduced in Barndorff-Nielsen (1977) and has been discussed by many authors since, particularly in connection with applications in finance. Some examples are Barndorff-Nielsen (1979), Barndorff-Nielsen and Blæsild (1981), Eberlein and Keller (1995), Prause (1999), Bibby and Sørensen (2003), Mencía and Sentana (2004) and McNeil et al. (2005).

The first four moments and skewness and kurtosis of the generalized hyperbolic $(\mathrm{GH})$ distribution were given by Barndorff-Nielsen and Blæsild (1981). The expressions are complicated and the reference rather difficult to obtain.

In this paper we establish a recursive method to determine the moments of the GH distribution of any order about the location parameter $\mu$ of the distribution. Then moments about any location, including raw moments and central moments can be obtained using well-known methods. This approach is easily implemented in software to enable the numeric evaluation of moments of any order about any location. An implementation is available in the R package HyperbolicDist (see Scott (2009)).

The approach of the present paper is very straightforward and produces moments of any order in comparison to the expressions given in Barndorff-Nielsen and Blæsild (1981), which require great care if errors are to avoided.

The GH distribution includes many interesting distributions as special and limiting cases including the normal inverse Gaussian (NIG) distribution, the hyperbolic distribution, the normal distribution, the skew hyperbolic- $t$ (SHT), and the variance gamma (VG) distribution. A comprehensive examination of limiting distributions is given by Eberlein and von Hammerstein (2003). A discussion of special and limiting cases of the GH distribution may also be found in Chapter 9 of Paolella (2007).

The SHT and VG arise for special values of the parameters of the generalized hyperbolic distribution and we show that the recursive method may be used to obtain moments of any order about any location for these distributions. Moments of the skew hyperbolic- $t$ up to order 4 were previously considered in Aas and Hobæk Haff (2006), and for the variance gamma in Seneta (2004). Implementations of the moment calculations for the SHT and VG may be found in the R packages SkewHyperbolic (Scott and Grimson (2009)) and VarianceGamma (Scott and Dong (2009)) respectively.

Recently, Barndorff-Nielsen and Stelzer (2005) developed expressions for the moments (and absolute moments) of the GH distribution as the sum of an infinite series of the moments of the symmetric GH distribution. They used these expressions to obtain expressions for the moments (and absolute moments) of the GH distribution as the sum of infinite series involving gamma functions and Bessel functions. Although we do not obtain expressions for the absolute moments, our approach yields expressions for the moments of a GH distribution which require only finite sums of terms which involve Bessel functions.

\section{The Generalized Hyperbolic Distribution}

Definition 2.1 The generalized hyperbolic (GH) distribution is given by 


$$
\begin{aligned}
& \operatorname{gh}(x \mid \lambda, \alpha, \beta, \delta, \mu)=a(\lambda, \alpha, \beta, \delta, \mu)\left[\delta^{2}+(x-\mu)^{2}\right]^{\left(\lambda-\frac{1}{2}\right) / 2} \\
& \quad \times K_{\lambda-1 / 2}\left(\alpha \sqrt{\delta^{2}+(x-\mu)^{2}}\right) \exp [\beta(x-\mu)] \\
& \text { with } \quad a(\lambda, \alpha, \beta, \delta, \mu)=\left(\alpha^{2}-\beta^{2}\right)^{\lambda / 2} /\left[\sqrt{2 \pi} \alpha^{\lambda-1 / 2} \delta^{\lambda} K_{\lambda}\left(\delta \sqrt{\alpha^{2}-\beta^{2}}\right)\right],
\end{aligned}
$$

where $K_{\lambda}$ is the modified Bessel function of the third kind and $x \in \mathbb{R}$. The domain of variation of the parameters is $\mu, \alpha \in \mathbb{R}$, and

$$
\begin{aligned}
& \delta \geq 0,|\beta|<\alpha \text { if } \lambda>0, \\
& \delta>0,|\beta|<\alpha \text { if } \lambda=0, \\
& \delta>0,|\beta| \leq \alpha \text { if } \lambda<0 .
\end{aligned}
$$

If $X$ has the distribution given in equation (1), we write $X \sim \operatorname{gh}(\lambda, \alpha, \beta, \delta, \mu)$.

Different parameterizations have been proposed in the literature. The one above we will call the 1st parameterization or $(\alpha, \beta)$ parameterization. A useful discussion is given in Prause (1999) where three further parameterizations are presented:

2nd or $(\zeta, \rho)$ parameterization : $\quad \zeta=\delta \sqrt{\alpha^{2}-\beta^{2}}, \quad \rho=\beta / \alpha$,

3rd or $(\xi, \chi)$ parameterization : $\xi=(1+\zeta)^{-1 / 2}, \quad \chi=\xi \rho$,

4th or $(\bar{\alpha}, \bar{\beta})$ parameterization : $\bar{\alpha}=\alpha \delta, \bar{\beta}=\beta \delta$.

Note, that for symmetric distributions $\beta=\bar{\beta}=\rho=\chi=0$ holds.

Results are often most easily expressed in terms of parameters from these alternative parameterizations. We use values such as $\zeta$ without comment when considering the distribution $\operatorname{gh}(\lambda, \alpha, \beta, \delta, \mu)$. The assumption is that $\zeta=\delta \sqrt{\alpha^{2}-\beta^{2}}$.

Frequently, the most useful representation of the GH distribution is as a variance-mean mixture of the generalized inverse Gaussian (GIG) distribution.

Definition 2.2 The generalized inverse Gaussian (GIG) distribution is given by

$$
\begin{aligned}
& \operatorname{gig}(x \mid \lambda, \chi, \psi)=a(\lambda, \chi, \psi) \times x^{\lambda-1} \exp [-(\chi / x+\psi x) / 2] \\
& \text { with } a(\lambda, \chi, \psi)=(\psi / \chi)^{\lambda / 2}\left[2 \mathrm{~K}_{\lambda}(\sqrt{\chi \psi})\right]^{-1}
\end{aligned}
$$

where $K_{\lambda}$ is the modified Bessel function of the third kind and $x>0$. The domain of variation of the parameters is 


$$
\begin{array}{r}
\lambda \in \mathbb{R} \text { if } \quad \chi>0 \text { and } \psi>0, \\
\lambda>0, \quad \psi>0 \text { if } \chi=0, \\
\lambda<0, \quad \chi>0 \text { if } \psi=0 .
\end{array}
$$

If $X$ has the distribution given in equation (4), we write $X \sim \operatorname{gig}(\lambda, \chi, \psi)$.

From Paolella (2007), we note that the case (5) is the normal or non-boundary case of the GIG. When the parameters satisfy (6), the GIG distribution becomes a gamma with shape parameter $\lambda$ and rate parameter $\psi / 2$. When the parameters satisfy (7), the GIG distribution becomes an inverse gamma with shape parameter $-\lambda$ and scale parameter $\chi / 2$.

The representation we will use to obtain our results can now be stated. If

$$
W \sim \operatorname{gig}\left(\lambda, \delta^{2}, \alpha^{2}-\beta^{2}\right) \quad \text { and } \quad X \mid W \sim \mathrm{N}(\mu+\beta W, W)
$$

then $X \sim \operatorname{gh}(\lambda, \alpha, \beta, \delta, \mu)$. For this result, which is originally due to Barndorff-Nielsen, see Paolella (2007), or Bibby and Sørensen (2003). Suppose $Z \sim \mathrm{N}(0,1)$ independently of $W \sim$ $\operatorname{gig}\left(\lambda, \delta^{2}, \alpha^{2}-\beta^{2}\right)$, then (8) implies that

$$
\mu+\beta W+\sqrt{W} Z \sim \operatorname{gh}(\lambda, \alpha, \beta, \delta, \mu),
$$

which expression is commonly used to simulate from the GH distribution.

We will need the moments of the GIG distribution, including the boundary cases where it reduces to the gamma or inverse gamma. From Paolella (2007) we have that if $X \sim \operatorname{gig}(\lambda, \chi, \psi)$ then provided $\chi>0$ and $\psi>0$,

$$
\mathrm{E}\left(X^{k}\right)=(\chi / \psi)^{(\lambda+k) / 2} K_{\lambda+k}(\sqrt{\chi \psi)}) / K_{\lambda}(\sqrt{\chi \psi)}) .
$$

When $\chi=0$ and $\lambda>0, X$ has a gamma distribution, and the moments are

$$
\mathrm{E}\left(X^{k}\right)=(2 / \psi)^{k} \Gamma(\lambda+k) / \Gamma(\lambda) .
$$

When $\psi=0$ and $\lambda<0, X$ has an inverse gamma distribution, and the moments are

$$
\mathrm{E}\left(X^{k}\right)=(\chi / 2)^{k} \Gamma(-\lambda-k) / \Gamma(-\lambda),
$$

provided $-\lambda>k$.

\section{Moments of the Generalized Hyperbolic}

Denote the $k$-th moment about $\mu$ by

$$
\overline{\mathrm{M}}_{k}=\mathrm{E}(X-\mu)^{k} .
$$

Then the mixture representation of the GH distribution leads to the following result for $\overline{\mathrm{M}}_{k}$.

Theorem 3.1 Suppose $X \sim \operatorname{gh}(\lambda, \alpha, \beta, \delta, \mu)$. Then

$$
\overline{\mathrm{M}}_{k}=\sum_{\ell=\lfloor(k+1) / 2\rfloor}^{k} \frac{k !}{(k-\ell) !(2 \ell-k) ! 2^{k-\ell}} \beta^{2 \ell-k} \mathrm{E}\left(W^{\ell}\right)
$$

where $W \sim \operatorname{gig}\left(\lambda, \delta^{2}, \alpha^{2}-\beta^{2}\right)$. 
Proof From (8), if $Z \sim \mathrm{N}(0,1)$ independently of $W \sim \operatorname{gig}\left(\lambda, \delta^{2}, \alpha^{2}-\beta^{2}\right)$, then

$$
\begin{aligned}
\overline{\mathrm{M}}_{k}= & =\mathrm{E}\left[(X-\mu)^{k}\right] \\
& =\mathrm{E}\left[(\beta W+\sqrt{W} Z)^{k}\right] \\
& =\sum_{i=0}^{k}\left(\begin{array}{c}
k \\
i
\end{array}\right) \beta^{k-i} \mathrm{E}\left(Z^{i}\right) \mathrm{E}\left(W^{k-(i / 2)}\right) \\
& =\sum_{j=0}^{\lfloor k / 2\rfloor}\left(\begin{array}{c}
k \\
2 j
\end{array}\right) \beta^{k-2 j} \frac{(2 j) !}{2^{j} j !} \mathrm{E}\left(W^{k-j}\right) \\
& =\sum_{j=0}^{\lfloor k / 2\rfloor} \frac{k !}{j !(k-2 j) ! 2^{j}} \beta^{k-2 j} \mathrm{E}\left(W^{k-j}\right) \\
& =\sum_{\lfloor\ell=(k+1) / 2\rfloor}^{k} \frac{k !}{(k-\ell) !(2 \ell-k) ! 2^{k-\ell}} \beta^{2 \ell-k} \mathrm{E}\left(W^{k}\right),
\end{aligned}
$$

where we have used the well-known result for the moments of the standard normal distribution, that

$$
\mathrm{E}\left(Z^{i}\right)= \begin{cases}0 & i \text { odd } \\ i !\left[2^{i / 2}(i / 2) !\right]^{-1} & i \text { even }\end{cases}
$$

The moments of the mixing distribution $W$ can be found from the moments of the GIG distribution, equation (10).

Lemma 3.2 Suppose $X \sim \operatorname{gh}(\lambda, \alpha, \beta, \delta, \mu)$. Then for $W$ as in Theorem 3.1

$$
\mathrm{E}\left(W^{k}\right)=\left(\delta^{2} / \zeta\right)^{\lambda+k} K_{\lambda+k}(\zeta) / K_{\lambda}(\zeta)
$$

provided $\chi>0$ and $\psi>0$.

Proof The mixing distribution $W$ is GIG with $\chi=\delta^{2}$ and $\psi=\alpha^{2}-\beta^{2}$. The result follows from substitution of these values in (10), noting that $\zeta=\delta \sqrt{\alpha^{2}-\beta^{2}}$.

Combining the result of Lemma 3.2 with Theorem 3.1 gives us the following result.

Corollary 3.1 Under the conditions of Theorem 3.1,

$$
\overline{\mathrm{M}}_{k}=\sum_{\ell=\lfloor(k+1) / 2\rfloor}^{k} \frac{k !}{(k-\ell) !(2 \ell-k) ! 2^{k-\ell}} \beta^{2 \ell-k}\left(\delta^{2} / \zeta\right)^{\lambda+k} K_{\lambda+k}(\zeta) / K_{\lambda}(\zeta)
$$

provided $\chi>0$ and $\psi>0$.

If $\beta=0$ the generalized hyperbolic distribution is symmetric and the moments take a particularly simple form. Note that the mean is just $\mu$ in this case and the moments about $\mu$ are the usual central moments.

Corollary 3.2 Suppose $X \sim \operatorname{gh}(\lambda, \alpha, 0, \delta, \mu)$. Then for any integer $k>0, \overline{\mathrm{M}}_{k}$ is the $k$-th central moment, and $\overline{\mathrm{M}}_{k}=0$ for $k$ odd, while

$$
\begin{aligned}
\overline{\mathrm{M}}_{k} & =a_{k, k / 2}\left(\delta^{2} / \zeta\right)^{\lambda+k / 2} K_{\lambda+k / 2}(\zeta) / K_{\lambda}(\zeta) \\
& =k !\left[2^{k / 2}(k / 2) !\right]^{-1}\left(\delta^{2} / \zeta\right)^{\lambda+k / 2} K_{\lambda+k / 2}(\zeta) / K_{\lambda}(\zeta)
\end{aligned}
$$

for $k$ even. 
Corollary 3.1 gives an expression for the moments about $\mu$ of any order for the GH distribution. For implementation, it is useful to have a recursive method of obtaining the coefficients in the summation which appears in (14) and (16). Define the coefficients $a_{k, \ell}$ for $\ell=1,2, \ldots, k$ and $k=1,2, \ldots$ by

$$
a_{k, \ell}= \begin{cases}0 & \ell<\lfloor(k+1) / 2 \\ k !\left[(k-\ell) !(2 \ell-k) ! 2^{k-\ell}\right]^{-1} & \lfloor(k+1) / 2\rfloor \leq \ell \leq k .\end{cases}
$$

For convenience, define $m(k)=\lfloor(k+1) / 2\rfloor$.

Theorem 3.3 The coefficients $a_{k, \ell}$ may be determined recursively as $a_{1,1}=1$ and

$$
a_{k, \ell}=a_{k-1, \ell-1}+(2 \ell-k+1) a_{k-1, \ell} .
$$

Proof The result is true for $k=1$. We show it is true for all $k$ by induction.

Assume the result is true for $k-1$.

For $k$ even and $\ell=m(k)=k / 2$, the recurrence relation (18) gives

$$
\begin{aligned}
a_{k, m(k)} & =(2 m(k)-k+1) a_{k-1, m(k)} \\
& =(2 k / 2-k+1) a_{k-1, k / 2} \\
& =\frac{(k-1) !}{(k-1-k / 2) !(k-(k-1)) ! 2^{k-1-k / 2}} \\
& =\frac{2(k-k / 2)(k-1) !}{(k-k / 2) ! 1 ! 2^{k-k / 2}} \\
& =\frac{k !}{(k-m(k)) ! 0 ! 2^{k-m(k)}} \\
& =\frac{k !}{(k-m(k)) !(2 m(k)-k) ! 2^{k-m(k)}}
\end{aligned}
$$

as required.

For any other values of $k$ and $\ell$ we have

$$
\begin{aligned}
a_{k, \ell} & =a_{k-1, \ell-1}+(2 \ell-k+1) a_{k-1, \ell} \\
& =\frac{(k-1) !}{(k-1-\ell+1) !(2(\ell-1)-(k-1)) ! 2^{k-\ell}}+\frac{(2 \ell-k+1)(k-1) !}{(k-1-\ell) !(2 \ell-(k-1)) ! 2^{k-1-\ell}} \\
& =\frac{k !}{(k-\ell) !\left(2(\ell-k) ! 2^{k-\ell}\right.}\left[\frac{2 \ell-k}{k}+\frac{2(k-\ell)}{k}\right] \\
& =\frac{k !}{(k-\ell) !(2 \ell-k) ! 2^{k-\ell}}
\end{aligned}
$$

which is again of the required form, completing the proof.

Note: It is of course possible to obtain the moments about $\mu$ using the expression given in Corollary 3.1, rather than using the recursive calculation of the coefficients. The recursion is very stable numerically however and avoids the possibility of intermediate expression swell which can occur for the factorials present in equation (16). 
The coefficients $a_{k, \ell}$ for $k=1,2,3,4$ are

$$
\begin{aligned}
& a_{1,1}=1 \\
& a_{2,1}=1, \quad a_{2,2}=1 \\
& a_{3,1}=0, \quad a_{3,2}=3, \quad a_{3,3}=1 \\
& a_{4,1}=0, \quad a_{4,2}=3, \quad a_{4,3}=6, \quad a_{4,4}=1 .
\end{aligned}
$$

so the first four moments around $\mu$ are

$$
\begin{aligned}
& \overline{\mathrm{M}}_{1}=\left(\delta^{2} / \zeta\right) \beta K_{\lambda+1}(\zeta) / K_{\lambda}(\zeta) \\
& \overline{\mathrm{M}}_{2}=\left[\left(\delta^{2} / \zeta\right) K_{\lambda+1}(\zeta)+\left(\delta^{2} / \zeta\right)^{2} \beta^{2} K_{\lambda+2}(\zeta)\right] / K_{\lambda}(\zeta) \\
& \overline{\mathrm{M}}_{3}=\left[3\left(\delta^{2} / \zeta\right)^{2} \beta K_{\lambda+2}(\zeta)+\left(\delta^{2} / \zeta\right)^{3} \beta^{3} K_{\lambda+3}(\zeta)\right] / K_{\lambda}(\zeta) \\
& \overline{\mathrm{M}}_{4}=\left[3\left(\delta^{2} / \zeta\right)^{2} K_{\lambda+2}(\zeta)+6\left(\delta^{2} / \zeta\right)^{3} \beta^{2} K_{\lambda+3}(\zeta)+\left(\delta^{2} / \zeta\right)^{4} \beta^{4} K_{\lambda+4}(\zeta)\right] / K_{\lambda}(\zeta)
\end{aligned}
$$

We have seen that calculation of moments about $\mu$ is mathematically convenient for the GH distribution. Typically though, moments about zero (raw moments) or about the mean (central moments) are required. Changing the point about which the moments are calculated is straightforward however involving a simple application of the binomial theorem. This is well-known in the case of interchanging between raw and central moments, but the same approach works more generally.

Lemma 3.4 For any constants $a$ and $b$, and any positive integer $k$,

$$
\mathrm{E}\left[(X-b)^{k}\right]=\sum_{i=0}^{k}\left(\begin{array}{c}
k \\
i
\end{array}\right)(a-b)^{k-i} \mathrm{E}\left[(X-a)^{i}\right]
$$

\section{Proof}

$$
\begin{aligned}
\mathrm{E}\left[(X-b)^{k}\right] & =\mathrm{E}\left[(X-a-b+a)^{k}\right] \\
& =\mathrm{E}\left[\sum_{i=0}^{k}\left(\begin{array}{c}
k \\
i
\end{array}\right)(a-b)^{k-i}(X-a)^{i}\right] \\
& =\sum_{i=0}^{k}\left(\begin{array}{c}
k \\
i
\end{array}\right)(a-b)^{k-i} \mathrm{E}\left[(X-a)^{i}\right] .
\end{aligned}
$$

To calculate the $k$-th moment of the GH distribution about any real $a$ for any integer $k$, the procedure is straightforward. Calculate the coefficients $a_{k, \ell}$ for all $\ell$ for that value of $k$ using the recursion (18) and then the moments around $\mu$ up to the $k$-th using (16). Finally use (20) to obtain the $k$-th moment about $a$ from the moments around $\mu$. This procedure has been programmed by the authors in $\mathrm{R}$ and is included in the package HyperbolicDist (see Scott (2009)) available on CRAN. Numerical comparisons were carried out for moments up to the 50th with the use of numerical integration to determine the moments and showed good 
agreement. In the package HyperbolicDist, the function momRecursion calculates the coefficients $a_{k, \ell}$, gigMom calculates moments for the GIG distribution, and ghypMom uses these functions and momChangeAbout to calculate moments of any order about any location for the GH distribution.

The procedure described above can be compared to the results given in Section 3 of BarndorffNielsen and Stelzer (2005). In the present paper no expressions are obtained for absolute moments, but our methods are much more convenient in determining moments than those given by Barndorff-Nielsen and Steltzer.

\section{Low Order Moments}

Interest in moments commonly is focused on low order moments, up to order four, and the skewness and kurtosis. It is useful to define $R_{\lambda, i}(\zeta)$ for $i$ a positive integer according to

$$
R_{\lambda, i}(\zeta)=K_{\lambda+i}(\zeta) / K_{\lambda}(\zeta)
$$

Besides leading to compact expressions, the use of this ratio of Bessel functions has computational advantages. When the argument $\zeta$ is large, the Bessel function $K_{\lambda}(\zeta)$ becomes very small and underflow can result. One solution (implemented in the R function besselK) is to use an exponentially scaled version of $K_{\lambda}(\zeta)$, which means calculating $e^{\zeta} K_{\lambda}(\zeta)$ instead of the Bessel function itself. If the exponentially scaled Bessel function is calculated for the both the numerator and denominator when calculating $R_{\lambda, i}(\zeta)$ then an accurate value of the ratio $R_{\lambda, i}(\zeta)$ can be obtained. In the package HyperbolicDist, the function besselRatio implements this approach allowing accurate computation even for very large values of $\zeta$.

Denote the central moments by $\mathrm{M}_{k}=\mathrm{E}\left[(X-\mathrm{E} X)^{k}\right]$ for $k=2,3, \ldots$ Using the results of the previous section we can obtain expressions for the mean, $\mathrm{M}_{2}, \mathrm{M}_{3}$, and $\mathrm{M}_{4}$. We have

$$
\begin{gathered}
\mathrm{E} X=\mu+\frac{\beta \delta}{\sqrt{\alpha^{2}-\beta^{2}}} R_{\lambda, 1}(\zeta)=\mu+\beta \delta^{2} \zeta^{-1} R_{\lambda, 1}(\zeta) \\
\mathrm{M}_{2}=\operatorname{Var}(X)=\delta^{4} \beta^{2} \zeta^{-2}\left[R_{\lambda, 2}(\zeta)-R_{\lambda, 1}^{2}(\zeta)\right]+\delta^{2} \zeta^{-1} R_{\lambda, 1}(\zeta) \\
\mathrm{M}_{3}=\delta^{6} \beta^{3} \zeta^{-3}\left[R_{\lambda, 3}(\zeta)-3 R_{\lambda, 2}(\zeta) R_{\lambda, 1}(\zeta)+2 R_{\lambda, 1}^{3}(\zeta)\right]+3 \delta^{4} \beta \zeta^{-2}\left[R_{\lambda, 2}(\zeta)-R_{\lambda, 1}^{2}(\zeta)\right]
\end{gathered}
$$

and

$$
\begin{aligned}
\mathrm{M}_{4}= & \delta^{8} \beta^{4} \zeta^{-4}\left[R_{\lambda, 4}(\zeta)-4 R_{\lambda, 3}(\zeta) R_{\lambda, 1}(\zeta)+6 R_{\lambda, 2}(\zeta) R_{\lambda, 1}^{2}(\zeta)-3 R_{\lambda, 1}^{3}(\zeta)\right] \\
& +6 \delta^{6} \beta^{2} \zeta^{-3}\left[R_{\lambda, 3}(\zeta)-2 R_{\lambda, 2}(\zeta) R_{\lambda, 1}(\zeta)+R_{\lambda, 1}^{3}(\zeta)\right] .
\end{aligned}
$$

The expressions for the mean and variance of the GH distribution are given in Prause (1999) or Bibby and Sørensen (2003). Expressions for the third and fourth moments were also given in Barndorff-Nielsen and Blæsild (1981). These expressions are sufficiently complicated that to our knowledge no authors have subsequently reproduced them in a paper. 


\section{$5 \quad$ Related Distributions}

A number of special and limiting cases of the GH distribution have been investigated in the literature. Special cases are the hyperbolic and normal inverse Gaussian distributions. Limiting cases of interest are the skew hyperbolic- $t$ and the variance gamma.

The hyperbolic distribution is the particular case of $\lambda=1$. There appear to be no simplifications in the moment results for this case: simply set $\lambda=1$ in the results for the GH distribution.

\subsection{The Normal Inverse Gaussian Distribution}

Definition 5.1 The normal inverse Gaussian (NIG) distribution is given by

$$
\begin{gathered}
\operatorname{nig}(x \mid \alpha, \beta, \delta, \mu)=a(\alpha, \beta, \delta, \mu)\left[\delta^{2}+(x-\mu)^{2}\right]^{-1 / 2} \\
\quad \times K_{1}\left(\alpha \sqrt{\delta^{2}+(x-\mu)^{2}}\right) \exp [\beta(x-\mu)] \\
\text { with } \quad a(\alpha, \beta, \delta, \mu)=\pi^{-1} \delta \alpha \exp \left(\delta \sqrt{\alpha^{2}-\beta^{2}}\right) .
\end{gathered}
$$

The domain of variation of the parameters is $\mu \in \mathbb{R}, \delta>0$ and $0 \leq|\beta|<\alpha$.

The NIG is the special case of the GH distribution when $\lambda=-1 / 2$. The density function given as (26) is readily obtained from the density (1) via the well-known properties of the modified Bessel function (see Barndorff-Nielsen and Blæsild (1981) for example):

$$
K_{1 / 2}(x)=2^{-1 / 2} \sqrt{\pi} x^{-1 / 2} \exp (-x) \quad \text { and } \quad K_{-\lambda}(x)=K_{\lambda}(x) .
$$

The cumulant generating function of the NIG was given by Rydberg (1997). Simple expressions were given for the first four cumulants, the skewness and the kurtosis. These results reappear in later works - see Barndorff-Nielsen (1998), Barndorff-Nielsen and Prause (2001), Bibby and Sørensen (2003) and Barndorff-Nielsen et al. (2004). The results of Section 3 specialized to the case $\lambda=-1 / 2$ enable the moments to be found by a different route using the well-known simplification of the modified Bessel function when the index is of the form $n+1 / 2$ with $n$ an integer:

$$
K_{n+1 / 2}(x)=2^{-1 / 2} \sqrt{\pi} x^{-1 / 2} e^{-x}\left(1+\sum_{i=1}^{n} \frac{(n+i) !}{i !(n-i) !} 2^{-i} x^{-i}\right) .
$$

See for example Jørgensen (1982), p.170. Note also that the term outside the brackets on the right hand side of this equality is actually $K_{1 / 2}(x)$.

For the NIG, there is no apparent simplification in the expression for the moments over what is given in Section 3, but equation (27) enables the Bessel function values required to be easily calculated.

Expressions for the first four moments about $\mu$ can be obtained but are somewhat complicated. We have

$$
\begin{gathered}
\overline{\mathrm{M}}_{1}=\delta^{2} \beta \zeta^{-1} \\
\overline{\mathrm{M}}_{2}=\beta^{2} \delta^{4}\left(\zeta^{-2}+\zeta^{-3}\right)+\delta^{2} \zeta^{-1}
\end{gathered}
$$




$$
\overline{\mathrm{M}}_{3}=3 \delta^{4} \beta\left(\zeta^{-1}+\zeta^{-3}\right)+\delta^{6} \beta^{3}\left(\zeta^{-2}+3 \zeta^{-4}+3 \zeta^{-5}\right)
$$

and

$$
\overline{\mathrm{M}}_{4}=3 \delta^{4}\left(\zeta^{-2}+\zeta^{-3}\right)+6 \delta^{6} \beta^{2}\left(\zeta^{-3}+3 \zeta^{-4}+3 \zeta^{-5}\right)+\delta^{8} \beta^{4}\left(\zeta^{-4}+6 \zeta^{-5}+15 \zeta^{-6}+15 \zeta^{-7}\right) .
$$

After some algebra it can be shown that

$$
\begin{gathered}
\mathrm{E}(X)=\mu+\delta^{2} \beta \zeta^{-1}, \\
\operatorname{Var}(X)=\delta^{4} \alpha^{2} \zeta^{-3}, \\
\mathrm{M}_{3}=3 \delta^{6} \alpha^{2} \beta \zeta^{-5},
\end{gathered}
$$

and

$$
\mathrm{M}_{4}=3 \delta^{8} \alpha^{2}\left(\alpha^{2}+\beta^{2}\right) \zeta^{-7} .
$$

These results agree with those given by Rydberg (1997) and other authors.

\subsection{The Skew Hyperbolic $t$ Distribution}

The skew hyperbolic $t$ (SHT) distribution is a limiting case of the GH distribution obtained when $\lambda=-\nu / 2$ and $\alpha \rightarrow|\beta|$. It is of interest in financial applications but has had less investigation than the hyperbolic and NIG. The SHT is almost as analytically tractable as the NIG distribution, and it allows for very heavy tails and substantial skewness. The special case of the symmetric Student- $t$ distribution is mentioned by Prause (1999) as a limit of the generalized hyperbolic with a reference to an unpublished report of Blæsild. Other references are BarndorffNielsen and Shephard (2001), Jones and Faddy (2003), Mencía and Sentana (2004), Demarta and McNeil (2005), Aas and Hobæk Haff (2005), and Aas and Hobæk Haff (2006). The most extensive discussion is in the last-mentioned paper. A number of proposals have been presented for generalizing the Student- $t$ distribution to produce a skewed distribution and we could just call the SHT the skewed Student- $t$ distribution. The name we have chosen to use reflects that this is the form of a skewed Student- $t$ distribution which arises from the generalized hyperbolic distribution. Note that other names are used by different authors. Aas and Hobæk Haff (2006) use the name generalized hyperbolic skew Student's t-distribution. Paolella (2007) calls the distribution the asymmetric hyperbolic $t$.

To evaluate the moments we use a well-known result (see for example Abramowitz and Stegun (1972)) concerning the limiting behaviour of the Bessel function near zero:

$$
K_{\nu}(x) \sim \Gamma(\nu) 2^{\nu-1} x^{-\nu} \text { for } \quad x \rightarrow 0, \nu>0 .
$$

Note also that $K_{\nu}(x)=K_{-\nu}(x)$.

Definition 5.2 The skew hyperbolic $t$ (SHT) distribution is given by

$$
\operatorname{sht}(x \mid \nu, \beta, \delta, \mu)=\frac{\delta^{\nu}|\beta|^{(\nu+1) / 2} K_{(\nu+1) / 2}\left(\beta \sqrt{\delta^{2}+(x-\mu)^{2}}\right) \exp [\beta(x-\mu)]}{2^{(\nu-1) / 2} \Gamma(\nu / 2) \sqrt{\pi}\left[\sqrt{\delta^{2}+(x-\mu)^{2}}\right]^{(\nu+1) / 2}} .
$$

The domain of variation of the parameters is $\mu \in \mathbb{R}, \delta>0, \beta \in \mathbb{R}$, and $\nu>0$. If $X$ has the distribution given in equation (37), we write $X \sim \operatorname{sht}(\nu, \beta, \delta, \mu)$. 
The SHT has a representation as a mean-variance mixture just as the GH does. From Paolella (2007), if $W$ is inverse gamma with shape parameter $\nu / 2$ and scale parameter $\delta^{2} / 2$ then

$$
\mu+\beta W+\sqrt{W} Z \sim \operatorname{sht}(\nu, \beta, \delta, \mu) .
$$

Note that the inverse gamma is actually a limiting case or boundary case of the GIG, namely that $W$ is inverse gamma with shape parameter $\nu / 2$ and scale parameter $\delta^{2} / 2$ is the same as $W \sim \operatorname{gig}\left(-\nu / 2, \delta^{2}, 0\right)$ (see Paolella (2007)).

Because of the representation of the SHT as a mean-variance mixture, the same arguments used to obtain the moments of the GH distribution can be carried through for the SHT, with the moments of the GIG being replaced by the moments of the inverse gamma. From (12) we see that if $W$ is inverse gamma with shape parameter $\nu / 2$ and scale parameter $\delta^{2} / 2$

$$
\mathrm{E}\left(W^{k}\right)=\left(\delta^{2} / 2\right)^{k} \Gamma(\nu / 2-k) / \Gamma(\nu / 2)
$$

provided $\nu>2 k$. Then for this boundary case (16) becomes

$$
\overline{\mathrm{M}}_{k}=\sum_{\ell=m(k)}^{k} a_{k, \ell} \delta^{2 \ell} \beta^{2 \ell-k} \frac{\Gamma(\nu / 2-\ell)}{\Gamma(\nu / 2) 2^{\ell}}
$$

provided $\nu>2 k$.

This approach has been implemented in the package SkewHyperbolic available from CRAN, see Scott and Grimson (2009). The function to calculate moments of the SHT distribution is called skewhypMom.

For the first four moments about $\mu$ we see that

$$
\begin{gathered}
\overline{\mathrm{M}}_{1}=\frac{\delta^{2} \beta}{\nu-2}, \quad \text { when } \quad \nu>2, \\
\overline{\mathrm{M}}_{2}=\frac{\delta^{4} \beta^{2}}{(\nu-2)(\nu-4)}+\frac{\delta^{2}}{\nu-2}, \quad \text { when } \quad \nu>4, \\
\overline{\mathrm{M}}_{3}=\frac{\delta^{6} \beta^{3}}{(\nu-2)(\nu-4)(\nu-6)}+\frac{3 \delta^{4} \beta}{(\nu-2)(\nu-4)}, \quad \text { when } \quad \nu>6,
\end{gathered}
$$

and

$$
\begin{aligned}
\overline{\mathrm{M}}_{4}= & \frac{\delta^{8} \beta^{4}}{(\nu-2)(\nu-4)(\nu-6)(\nu-8)}+\frac{6 \delta^{6} \beta^{2}}{(\nu-2)(\nu-4)(\nu-6)}+\frac{3 \delta^{4}}{(\nu-2)(\nu-4)}, \\
& \text { when } \quad \nu>8 .
\end{aligned}
$$

The mean of the SHT is then

$$
\mathrm{E}(X)=\mu+\frac{\delta^{2} \beta}{(\nu-2)}, \quad \text { when } \quad \nu>2,
$$

which allows us to calculate the central moments using

$$
\mathrm{M}_{k}=\sum_{i=0}^{k}\left(\begin{array}{l}
k \\
i
\end{array}\right)\left(-\frac{\delta^{2} \beta}{(\nu-2)}\right)^{k-i} \overline{\mathrm{M}}_{i}, \quad \text { when } \quad \nu>2 k .
$$


The second, third and fourth central moments are respectively

$$
\begin{aligned}
\mathrm{M}_{2}= & \frac{2 \delta^{4} \beta^{2}}{(\nu-2)^{2}(\nu-4)}+\frac{\delta^{2}}{\nu-2}, \text { when } \nu>4, \\
\mathrm{M}_{3}= & \frac{16 \delta^{6} \beta^{3}}{(\nu-2)^{3}(\nu-4)(\nu-6)}+\frac{6 \delta^{4} \beta}{(\nu-2)^{2}(\nu-4)}, \\
& \text { when } \quad \nu>6,
\end{aligned}
$$

and

$$
\begin{aligned}
\mathrm{M}_{4}= & \frac{12 \delta^{8} \beta^{4}(\nu+10)}{(\nu-2)^{4}(\nu-4)(\nu-6)(n u-8)}+\frac{12 \delta^{6} \beta^{2}(\nu+2)}{(\nu-2)^{3}(\nu-4)(\nu-6)}+\frac{3 \delta^{4}}{(\nu-2)(\nu-4)}, \\
& \text { when } \quad \nu>8
\end{aligned}
$$

The ordinary central Student- $t$ distribution is a further specialization of the SHT to the case where $\beta \rightarrow 0$ and $\delta=\sqrt{\nu}$, see Aas and Hobæk Haff (2005). Then the moments around $\mu$ are moments around 0 and are the central moments as well.

From the expression above for the moments around $\mu$ for the SHT, we see that $\overline{\mathrm{M}}_{k}=0$ for $k$ odd, while for $k$ even,

$$
\overline{\mathrm{M}}_{k}=\frac{k !}{2^{k / 2}(k / 2) !} \frac{\nu^{k / 2}}{(\nu / 2-1)(\nu / 2-2) \ldots(\nu / 2-k / 2)}, \quad \text { when } \quad \nu>2 k .
$$

A straightforward induction argument can be used to show this is expression is the same as

$$
\nu^{k / 2} \frac{\Gamma((k+1) / 2) \Gamma((\nu-k) / 2)}{\Gamma(1 / 2) \Gamma(\nu / 2)} \text { when } \quad \nu>2 k,
$$

which is the form in which the moments of the $t$ distribution are given in Kendall and Stuart (1969), p.375.

\subsection{The Variance Gamma Distribution}

The variance gamma distribution is another limiting case of the the GH distribution which arises when $\delta=0$. The variance-gamma distribution was introduced by Madan and Seneta in Madan and Seneta (1990), but had actually appeared much earlier in a different guise, as the Bessel function distribution, see McKay (1932). It has been examined in detail recently in yet another guise as the generalized asymmetric Laplace distribution in Kotz et al. (2001).

Definition 5.3 The variance gamma (VG) distribution is given by

$$
\operatorname{vg}(x \mid c, \sigma, \theta, \nu)=\alpha(c, \sigma, \theta, \nu) \exp \left[\theta(x-c) / \sigma^{2}\right]|x-c|^{1 / \nu-1 / 2} \mathrm{~K}_{1 / \nu-1 / 2}\left(\sigma^{-2}|x-c| \sqrt{2 \sigma^{2} / \nu+\theta^{2}}\right)
$$

with $\alpha(\theta, \nu, \sigma, c)=2\left[\sqrt{2 \sigma^{2} / \nu+\theta^{2}}\right]^{1 / 2-1 / \nu} /\left[\sigma \sqrt{2 \pi} \nu^{1 / \nu} \Gamma(1 / \nu)\right]$,

the domain of the parameters being

$$
\nu>0, \quad \sigma>0, \quad-\infty<\theta<\infty, \quad \text { and }-\infty<c<\infty .
$$

If $X$ has the distribution given in equation (51), we write $X \sim \operatorname{vg}(c, \sigma, \theta, \nu)$. 
Paolella (2007) shows the VG distribution is a limiting case of the GH when $\delta=0$. The parameters of the VG and the GH distribution are related by $\mu=c, \alpha=\left(\sqrt{2 \sigma^{2} / \nu+\theta^{2}}\right) / \sigma^{2}$, $\beta=\theta / \sigma^{2}$, and $\lambda=1 / \nu$.

The VG has a representation as a mean-variance mixture. From Paolella (2007), if $W$ is gamma with shape parameter $\lambda$ and rate parameter $\left(\alpha^{2}-\beta^{2}\right) / 2$ then

$$
\mu+\beta W+\sqrt{W} Z \sim \operatorname{vg}(c, \sigma, \theta, \nu) .
$$

Because of this representation we can obtain moments of the VG distribution by the argument used for the GH distribution, with the moments of the GIG being replaced by the moments of the gamma distribution. From equation (11) we see that if $W$ is gamma with shape parameter $\lambda$ and rate parameter $\left(\alpha^{2}-\beta^{2}\right) / 2$,

$$
\mathrm{E}\left(X^{k}\right)=\left[2 /\left(\alpha^{2}-\beta^{2}\right)\right]^{k} \Gamma(\lambda+k) / \Gamma(\lambda),
$$

and then (16) becomes

$$
\overline{\mathrm{M}}_{k}=\sum_{\ell=m(k)}^{k} a_{k, \ell} 2^{\ell} \beta^{2 \ell-k} \frac{\Gamma(\lambda+\ell)}{\Gamma(\lambda)\left(\alpha^{2}-\beta^{2}\right)^{\ell}} .
$$

The first four central moments plus the skewness and kurtosis were obtained in Seneta (2004). These can be obtained using the recursive method. For example the first two moments around $c$ are

$$
\overline{\mathrm{M}}_{1}=\theta
$$

and

$$
\overline{\mathrm{M}}_{2}=\sigma^{2}+\theta^{2}(\nu+1) .
$$

The central moments are then found from

$$
\mathrm{M}_{k}=\sum_{i=0}^{k}\left(\begin{array}{c}
k \\
i
\end{array}\right)(-\theta)^{k-i} \overline{\mathrm{M}}_{i} .
$$

This gives $\mathrm{E}(X)=c+\theta$, and $\operatorname{Var}(X)=\sigma^{2}+\nu \theta^{2}$. The first four central moments were previously given by Seneta $(2004)$.

\section{Acknowledgement}

DS is grateful for the financial assistance of Finance Online GmbH Zürich and for the hospitality of the Institute of Theoretical Physics, ETH Zürich, where much of this work was undertaken.

\section{References}

Kjersti Aas and Ingrid Hobæk Haff. NIG and skew Student's t: Two special cases of the generalised hyperbolic distribution. SAMBA/01/05, January 2005. SAMBA, Norwegian Computing Center. 
Kjersti Aas and Ingrid Hobæk Haff. The generalised hyperbolic skew student's $t$-distribution. Journal of Financial Econometrics, 4(2):275-309, 2006.

M. Abramowitz and I. Stegun. Handbook of Mathematical Functions with Formulas, Graphs, and Mathematical Tables. Dover, New York, 1972. 1046 p.

O. E. Barndorff-Nielsen. Exponentially decreasing distributions for the logarithm of particle size. Proceedings of the Royal Society of London A, 353:401-419, 1977.

O. E. Barndorff-Nielsen. Models for non-Gaussian variation; with applications to turbulence. Proc. Roy. Soc. London A, 868:501-520, 1979.

O. E. Barndorff-Nielsen. Processes of normal inverse Gaussian type. Finance and Stochastics, 2:41-68, 1998.

O. E. Barndorff-Nielsen and P. Blæsild. Hyperbolic distributions and ramifications: Contributions to theory and applications. In C. Taillie, G. P. Patil, and B. A. Baldessari, editors, Statistical distributions in scientific work, volume 4, pages 19-44. D. Reidel, Amsterdam, 1981.

O. E. Barndorff-Nielsen and K. Prause. Apparent scaling. Finance and Stochastics, 5:103-113, 2001.

O. E. Barndorff-Nielsen and N. Shephard. Normal modified stable processes. Theor. Probab. Math. Statist., 65:1-19, 2001.

O. E. Barndorff-Nielsen and R. Stelzer. Absolute moments of generalized hyperbolic distributions and approximate scaling of normal inverse Gaussian Lévy processes. Scandinavian Journal of Statistics, 32(4):617-637, 2005.

O. E. Barndorff-Nielsen, P. Blæsild, and J. Schmiegel. A parsimonious and universal description of turbulent velocity increments. European Physical Journal B, 41:345-363, 2004.

B. O. Bibby and M. Sørensen. Hyperbolic processes in finance. In S. T. Rachev, editor, Handbook of Heavy Tailed Distributions in Finance, pages 211-248. Elsevier Science B. V., 2003.

S. Demarta and A. J. McNeil. The t-copula and related copulas. International Statistical Review, 73(1):111-129, 2005.

E. Eberlein and E. A. von Hammerstein. Generalized hyperbolic and inverse Gaussian distributions: Limiting cases and approximation of processes. FDM Preprint 80, April 2003. University of Freiburg.

Ernst Eberlein and Ulrich Keller. Hyperbolic distributions in finance. Bernoulli, 1(3):281-299, Sep. 1995.

M. C. Jones and M. J. Faddy. A skew extension of the $t$ distribution, with applications. J. Royal Statist. Soc. B, 65:159-174, 2003.

B. Jørgensen. Statistical properties of the generalized inverse Gaussian distribution, volume 9 of Lecture Notes in Statistics. Springer, Heidelberg, 1982.

M. G. Kendall and A. Stuart. The Advanced Theory of Statistics, volume 1. Charles Griffin \& Company, London, 3 edition, 1969. 439 p.

Samuel Kotz, Tomasz J. Kozubowski, and Krzystof Podgórski. The Laplace Distribution and Generalizations. Birkhäuser, Boston, 2001. 349 p.

Dilip B. Madan and Eugene Seneta. The variance gamma (V.G.) model for share market returns. The Journal of Business, 63:511-524, 1990. 
A. T. McKay. A Bessel function distribution. Biometrika, 24:39-44, 1932.

Alexander J. McNeil, Rüdiger Frey, and Paul Embrechts. Quantitative Risk Management. Princeton Series in Finance. Princeton University Press, Princeton, NJ, 2005. 538 p.

F. J. Mencía and E. Sentana. Estimation and testing of dynamic models with generalised hyperbolic innovations. CMFI Working Paper 0411, 2004. Madrid, Spain.

Marc S. Paolella. Intermediate Probability: A Computational Approach. Wiley, Chichester, 2007. ISBN 978-0-470-02637-3.

K. Prause. The Generalized Hyperbolic Model: Estimation, Financial Derivatives, and Risk Measures. PhD thesis, Universität Freiburg, 1999.

T. H. Rydberg. The normal inverse Gaussian Lévy process: Simulation and approximation. Commun. Statist.-Stochastic Models, 34:887-910, 1997.

David J. Scott. HyperbolicDist, 2009. URL http://CRAN.R-project.org/package= HyperbolicDist. R package version 0.6-2.

David J. Scott and Christine Yang Dong. VarianceGamma, 2009. URL http://CRAN. R-project.org/package=VarianceGamma. R package version $0.2-1$.

David J. Scott and Fiona Grimson. SkewHyperbolic, 2009. URL http://CRAN.R-project.org/ package=SkewHyperbolic, $\mathrm{R}$ package version 0.1-2.

Eugene Seneta. Fitting the variance-gamma model to financial data. J. Appl. Probab., 41A: 177-187, 2004. 